\title{
Responsabilidad social universitaria en España: un estudio de casos
}

\author{
Aldeanueva Fernández, Ignacio* \\ Jiménez Quintero, José Antonio**
}

\section{Resumen}

La Responsabilidad Social es un elemento clave en la gestión de las organizaciones. El objetivo fundamental de este artículo es explicar la situación que, en la actualidad, presenta la Responsabilidad Social en el Sistema Universitario Español. Para ello, se emplea la metodología del análisis de casos en las siguientes universidades: Universidad de Santiago de Compostela, Universidad Internacional de Andalucía y Universidad de Málaga. Concretamente, un estudio de casos múltiple, en el que se formulan siete (7) proposiciones teóricas y treinta y cinco (35) preguntas de investigación. Los resultados obtenidos indican que las instituciones analizadas, representativas de las universidades españolas, son socialmente responsables, consideran a sus grupos de interés y emplean criterios de eficiencia y transparencia en el desarrollo de sus actividades. Se concluye, en términos generales, que la Universidad asume un compromiso con la sociedad, contribuyendo al progreso de la misma.

Palabras clave: Responsabilidad social, sistema universitario español, grupos de interés, gestión, análisis de casos.

\section{University Social Responsibility in Spain: Case Studies}

\begin{abstract}
Social responsibility is a key element in managing organizations. The fundamental objective of this article is to explain the current situation of social responsibility in the Spanish university system. To accomplish this, case analysis methodology is used at the following universities: the University of Santiago de Compostela, the International University of Andalucia and the University of Malaga. Specifically, this is a study of multiple cases in which seven (7) theoretical propositions and thirty-five (35)

Recibido: 28-10-12. Aceptado: 14-06-13

* Doctor y profesor de la Universidad de Málaga (España). Departamento de Economía y Administración de Empresas, e-mail: ialdeanuevaf@uma.es

** Doctor y catedrático de la Universidad de Málaga (España). Departamento de Economía y Administración de Empresas; e-mail: jajq@uma.es
\end{abstract}


questions are formulated. Results indicate that the institutions analyzed, representative of Spanish universities, are socially responsible, consider their interest groups and use criteria of efficiency and transparency in developing their activities. Conclusions, in general terms, are that the university takes on a commitment to society, contributing to its progress.

Keywords: Social responsibility, spanish university system, interest groups, management, case analysis.

\section{Introducción}

La Universidad, institución de educación superior por excelencia, no ha sido ajena a la relevancia que, en el conjunto de las organizaciones, ha adquirido en los últimos años, la responsabilidad social. Por ello, como señala Aldeanueva (2012), en el ámbito de la educación superior, la Responsabilidad Social de la Universidad o Responsabilidad Social Universitaria representa un pilar fundamental en la gestión de las universidades, ya que afecta tanto al ámbito interno de estas instituciones como a las relaciones que las mismas mantienen con el entorno.

La dimensión social de la educación superior, tema actual y relevante, pone de manifiesto el papel que las universidades y el resto de instituciones de educación superior deben asumir para avanzar en la recuperación económica y la cohesión social. Para garantizar dicha dimensión es necesario un compromiso con la equidad y la igualdad de oportunidades en el acceso a la educación superior; también, un modelo de gestión universitaria que permita canalizar, de manera adecuada, todas las estrategias diseñadas en relación con este tema.

Por consiguiente, promover la Responsabilidad Social Universitaria implica potenciar la contribución de las universidades al desarrollo del entorno y favore- cer el retorno del conocimiento a la sociedad. Ello supone que la responsabilidad social forme parte de la misión de la Universidad y que los objetivos sociales, al igual que los financieros y de aumento de valor, alcancen un carácter estratégico para la institución.

En España, las acciones desarrolladas en materia de responsabilidad social Universitaria son variadas. Cabe destacar, además de la elaboración y publicación de memorias de responsabilidad social, por parte de las universidades españolas, las actuaciones tan heterogéneas que han sido implantadas, ya que abarcan desde las adhesiones a iniciativas internacionales relevantes en el ámbito que se estudia, hasta proyectos o programas liderados por las propias universidades. Este auge de la responsabilidad social en la Universidad Española se fundamenta, de manera importante, en la denominada Estrategia Universidad 2015 (Ministerio de Ciencia e Innovación, 2009).

La preocupación por el papel de las universidades en el conjunto de la sociedad española queda reflejada en dicho plan, en el que se establece que es necesario un esfuerzo para mejorar el papel de dichas instituciones como motor de progreso y competitividad. Ello supone modernizar su gestión y reforzar su papel en la sociedad como eje impulsor de la economía productiva. Por tanto, las uni- 
versidades españolas deben desarrollar capacidades que contribuyan a mejorar las necesidades sociales y económicas del país. Todo ello pone de manifiesto la importancia, cada vez mayor, que va adquiriendo la Responsabilidad Social en el Sistema Universitario Español.

Al respecto, Luzón et al. (2006) señalan que es necesario dotar a la Universidad Española de la flexibilidad necesaria para que pueda cumplir las funciones que la sociedad le exige como servicio público. Por consiguiente, la Universidad Española tiene una responsabilidad de carácter social y no únicamente económica. Ello implica, que el legado cultural, la creación y el fortalecimiento de nuevos entornos de aprendizaje más dinámicos y flexibles, el impulso de programas de formación e innovación docente y la promoción de la movilidad estudiantil, son aspectos que la Universidad Española no puede ignorar en los tiempos actuales.

El objetivo principal de este trabajo de investigación es explicar la situación que, actualmente, presenta la Responsabilidad Social, abarcando el gran número de variables y dimensiones que ofrece en el Sistema Universitario Español. Se persigue, por consiguiente, conocer el nivel de relevancia que presenta la Responsabilidad Social en las estrategias, en las políticas, en los procedimientos y, en definitiva, en los sistemas de gestión de las universidades objeto de estudio. También, es importante valorar el grado de relación existente entre las acciones desarrolladas por las universidades que conforman la muestra de la investigación, en materia de Responsabilidad Social, y los compromisos adquiridos con las mismas.
Para alcanzar los objetivos planteados se empleó la metodología del análisis casos, que en esta investigación es múltiple, ya que se trabaja con tres de ellos. La investigación realizada es descriptiva, exploratoria y explicativa. Se seleccionan tres universidades españolas, que forman la muestra o unidades de análisis, en las que la Responsabilidad Social constituye un eje estratégico. Se trata de la Universidad de Santiago de Compostela, la Universidad Internacional de Andalucía y la Universidad de Málaga.

Los criterios para seleccionar la muestra son la ubicación geográfica de las universidades, la primera de ellas se encuentra en el norte de España y las otras en el sur; la antigüedad, ya que la primera se fundó hace más de cinco siglos y las restantes hace escasas décadas y, finalmente, el compromiso adquirido por estas instituciones, en el desarrollo de sus actividades y en su gestión, con la Responsabilidad Social.

La información se obtuvo mediante la realización de entrevistas presenciales con los máximos responsables, en materia de Responsabilidad Social, de las universidades estudiadas; también, con la revisión documental, tanto interna (memorias, informes, estudios internos, páginas web, archivos de imagen, entre otros) como externa (publicaciones especializadas, informes de organismos oficiales y de medios de comunicación, entre otros); finalmente, la observación directa, con estancias en las ciudades de Santiago de Compostela, Sevilla, Baeza, la Rábida y Málaga, valorando en las universidades, con los responsables de las mismas en temas de Responsabilidad Social, el fenómeno objeto de estudio. 


\section{Responsabilidad social: un análisis empírico}

Al analizar la literatura de organización de empresas (Martínez et al, 2012) en los últimos años, se puede apreciar, de manera evidente, la presencia de un concepto: la Responsabilidad Social. Aunque existen aportaciones en la década de los sesenta del siglo pasado (Schlusberg, 1969), es en la década de los ochenta cuando empieza a adquirir relevancia. Ello es debido, fundamentalmente, a los numerosos casos de corrupción cometidos por diversas empresas, como Enron, que originó en el año 2001 un escándalo en el mundo empresarial y generó incertidumbre en los inversores (Brito, 2007). Como consecuencia de ello, se empiezan a considerar en la gestión empresarial aspectos sociales, medioambientales y laborales, además de los tradicionales económicos.

En términos generales, la Responsabilidad Social es una forma de gestionar las organizaciones, ya sean empresas, universidades $u$ otras. Las diversas definiciones existentes sobre el citado concepto ponen de manifiesto lo indicado. Al respecto, Carroll (1999) señala que la Responsabilidad Social Empresarial abarca las expectativas económica, legal, ética y discrecional que la sociedad tiene de las organizaciones en un momento dado.

Para abarcar, en su totalidad, el concepto de Responsabilidad Social es necesario conocer el de grupos de interés. La definición más empleada es la propuesta por Freeman (1984), en la que indica que los grupos de interés o stakeholders son cualquier grupo o individuo que puede afectar o ser afectado por la consecución de los objetivos de la empresa. La relación que mantienen ambas partes (organización y grupos de interés) es significativa e interesante porque, a través de la misma, la organización puede transmitir los valores que la definen (Noland y Phillips, 2010).

Al respecto, Maon et al. (2010), establecen que los stakeholders influyen, de manera significativa, en las respuestas que la organización proporciona a las cuestiones sociales. En cualquier caso, la gestión de las relaciones con los stakeholders debe adoptarla la organización de manera voluntaria (Carroll y Shabana, 2010).

La consolidación de la Responsabilidad Social como materia objeto de estudio es una realidad contrastada, como demuestra la diversidad de trabajos realizados sobre la misma. Entre ellos, cabe destacar los siguientes: la industria farmacéutica (Urdaneta, 2008), la ética empresarial (Bañón et al, 2011), las tecnologías de la información (Jin y Drozdenko, 2010), el rendimiento financiero en los mercados emergentes (Aras et al., 2010), la ventaja competitiva (Porter y Kramer, 2006), entre otros.

Aunque la Responsabilidad Social se identifique, en la mayoría de los casos, con el ámbito empresarial, también presenta una relevancia considerable en ámbito de la educación superior. Éste es el que se analiza en el presente trabajo de investigación, siendo necesario hacer referencia al concepto de Responsabilidad Social de la Universidad o Responsabilidad Social Universitaria. En opinión de Jiménez (2008), es la capacidad que tiene la Universidad, de difundir y poner en 
práctica un conjunto de principios y valores generales y específicos, por medio de cuatro procesos considerados clave en la Universidad, como son la gestión, la docencia, la investigación y la extensión universitaria. Respondiendo socialmente así, ante la propia comunidad universitaria y el país donde está inserta.

Por consiguiente, la Responsabilidad Social Universitaria, supone, como señalan Siracusa et al. (2012), un desafío para las universidades, ya que deben formar profesionales comprometidos con la sociedad en la que desarrollen, en un futuro próximo, su trabajo. En definitiva, es clave promover y gestionar de manera eficiente la Responsabilidad Social en las universidades, ya que ello supone aumentar la contribución de las mismas al desarrollo económico y social de su entorno. Por ello, las universidades deben fomentar las relaciones con la sociedad, sin olvidar las clásicas funciones que tienen asignadas: enseñanza-aprendizaje e investigación, desarrollo tecnológico e innovación y, finalmente, la gestión.

Por último, indicar que aunque la Responsabilidad Social Universitaria pertenece a un ámbito muy específico, el de la educación superior, también la literatura especializada ofrece trabajos de investigación relevantes sobre este tema. Entre ellos, destaca el elaborado por Vallaeys (2008), que explora las posibilidades que ofrece la Responsabilidad Social en las universidades. Otros autores, como Matten y Moon (2008), se centran en exponer las diferencias existentes en el tratamiento concedido a la Responsabilidad Social en las universidades europeas y estadounidenses. Por su parte, Benneworth y Jongbloed (2010) profundizan en este tema desde la perspectiva de los grupos de interés.

\section{Responsabilidad Social Universitaria: análisis de casos}

A continuación, se realiza un estudio comparado de los casos, siguiendo las orientaciones propuestas por Yin (1989). Por tanto, una vez analizada la literatura previa, se formulan 7 proposiciones teóricas y, seguidamente, se vinculan con la información recabada, entre la que se incluyen 35 preguntas de investigación que respondieron los gestores de la responsabilidad social en las universidades objeto de estudio. Ello permite generar conclusiones sobre el tema estudiado.

Proposición teórica $n^{\circ}$ 1: la Universidad de Santiago de Compostela, la Universidad Internacional de Andalucía y la Universidad de Málaga son universidades socialmente responsables, ya que incluyen entre sus preocupaciones aspectos de indole social, medioambiental $y$ económica.

En este punto, se debe valorar si las universidades analizadas están comprometidas, de manera real y efectiva, con la Responsabilidad Social. En los casos expuestos, se puede constatar como las actividades docentes, investigadoras y de extensión universitaria realizadas por estas instituciones se orientan a satisfacer, en la medida de lo posible, las necesidades del entorno, lo que supone una gestión ética e inteligente de los impactos que la Universidad genera en dicho entorno. La información obtenida es coherente con planteamientos teóricos previos, como los de Jiménez (2008) o Vallaeys (2008), que destacan el compromiso social, el respeto al medio ambiente y el buen gobierno como 
ejes básicos de la Responsabilidad Social de la Universidad.

La transparencia en sus actuaciones es otro elemento que caracteriza a las universidades analizadas. Ello está en consonancia con la rendición de cuentas de sus actividades a la sociedad, en el marco de la gobernanza, tal y como establece la Estrategia Universidad 2015 (Ministerio de Ciencia e Innovación, 2009). Por último, comentar que la Universidad de Santiago de Compostela, la Universidad Internacional de Andalucía y la Universidad de Málaga definen estrategias orientadas a todos sus grupos de interés teniendo en cuenta sus demandas y expectativas (Maon et al., 2010), lo que constituye un pilar básico en su compromiso con la Responsabilidad Social.

Proposición teórica $n^{\circ} 2$ : el diálogo permanente con la sociedad en su conjunto, por parte de la Universidad de Santiago de Compostela, de la Universidad Internacional de Andalucía y de la Universidad de Málaga, es un elemento diferenciador de las instituciones objeto de análisis, demostrando así su compromiso con la sociedad y constituyendo la Responsabilidad Social un importante instrumento para canalizar dicho compromiso.

La Universidad se legitima socialmente en la medida que responde a las necesidades sociales (De la Red, 2009). Dicha afirmación, pone de manifiesto la importancia que supone, en materia de Responsabilidad Social Universitaria, la colaboración, por parte de las instituciones de educación superior, con la sociedad. Las universidades analizadas, a través de diversos órganos colegiados, mantienen una interacción constante con la sociedad, lo que refuerza lo expresado por diversos autores especializados en la materia, como Días (2008), que indica que las universidades deben tener una conciencia social y contribuir con la sociedad a solucionar los problemas que afectan a la colectividad.

La relación que tienen las universidades estudiadas con la sociedad está estrechamente vinculada con la dimensión social del Espacio Europeo de Educación Superior, ya que un objetivo establecido, referido a la citada dimensión, es mejorar la interacción de las universidades con las organizaciones de la sociedad civil para incrementar su nivel de respuesta a las demandas sociales. Dicho compromiso con la sociedad está en consonancia con lo expuesto en la Declaración de Glasgow, en el año 2005, que señala que las universidades deben contribuir al bienestar social y estar abiertas al trabajo con la sociedad (EUA, 2005). En la misma línea, la Declaración de Lisboa establece que las universidades deben ayudar a la sociedad a hacer frente a los retos actuales y futuros (EUA, 2007).

En definitiva, los acuerdos de colaboración, con entidades públicas y privadas, por parte de la Universidad de Santiago de Compostela, la Universidad Internacional de Andalucía y la Universidad de Málaga refuerzan su compromiso social, ya que, como señala Husted (2000), el carácter dinámico de la Responsabilidad Social motiva que su evolución mantenga una estrecha relación con los intereses que la sociedad considera más justos en cada momento.

Proposición teórica $n^{\circ}$ 3: los efectos que generan la Universidad de Santiago de Compostela, la Universidad Internacional de Andalucía y la Universidad de 
Málaga al realizar sus actividades son menos perjudiciales para el medio ambiente que los que causaban antes de incorporar la Responsabilidad Social a sus respectivos sistemas de gestión.

Las diversas actuaciones en materia medioambiental, que llevan a cabo las universidades analizadas, son significativas y demuestran que dichas instituciones son socialmente responsables. Las universidades, al potenciar el uso eficiente y sostenible de los recursos que emplean, contribuyen a la preservación del medio ambiente.

Una de las características fundamentales que definen la educación para el desarrollo sostenible, concepto estrechamente relacionado con la Responsabilidad Social, es el aumento de la responsabilidad ambiental (UNESCO, 2007). Además, diversos proyectos internacionales, relativos a la sostenibilidad en la alta educación, inciden en la importancia que presenta esta materia en la gestión de las universidades. De acuerdo con la literatura, se puede citar el Instrumento de Medida para la Sostenibilidad de la Alta Educación (Proyecto AISHE), que señala como criterio fundamental la gestión medioambiental (DHO, 2007), o el New Jersey Higher Education Partnership for Sustainability, al indicar que el compromiso con la eficiencia energética, por parte de las instituciones de educación superior, contribuye tanto a la mejora del medio ambiente como a favorecer la sostenibilidad (NJHEPS, 2004).

En relación con las certificaciones medioambientales, cabe destacar, que varios centros de la Universidad de Santiago de Compostela se han certificado conforme a la Norma UNE-EN ISO 14001 y que la Universidad de Málaga se encuentra finalizando el proceso de certificación, en dicho estándar internacional de gestión ambiental, de la totalidad de su campus universitario. Ello demuestra un compromiso firme con el medio ambiente, por parte de estas dos universidades, al igual que más de cien mil organizaciones en todo el mundo que tienen certificado, según esta norma internacional, su sistema de gestión ambiental (Schwartz y Tilling, 2009).

Proposición teórica $n^{\circ}$ 4: la Universidad de Santiago de Compostela, la Universidad Internacional de Andalucía y la Universidad de Málaga han empleado, desde que se comprometieron con las políticas de Responsabilidad Social, de una manera más responsable los recursos económicos disponibles.

Aunque la Responsabilidad Social considere aspectos sociales y medioambientales en la gestión de las organizaciones, los económicos siguen siendo fundamentales. Dicha consideración es clave en las instituciones de educación superior, al igual que en las empresas, porque la Universidad, aunque presenta un carácter peculiar en el ámbito organizacional, debe generar recursos económicos que contribuyan a su correcta financiación; también, emplear adecuadamente los que tenga disponibles. Si esto no sucede, no podrá estar comprometida, de manera real, con el avance de la sociedad.

Por ello, la Universidad de Santiago de Compostela, la Universidad Internacional de Andalucía y la Universidad de Málaga supervisan y controlan su desempeño económico-financiero, lo que supone un firme compromiso con la rendición de cuentas. Ello supone ser responsa- 
bles ante sus grupos de interés y proporcionarles la información necesaria (Benneworth y Jongbloed, 2010).

Además, estas universidades, en relación con sus datos económicos, son transparentes, ya que los mismos son evaluados por entidades independientes. Ello está en consonancia con lo establecido en la Conferencia Mundial sobre la Educación Superior, celebrada en París en el año 2009, en la que se fijaban como objetivos fundamentales alcanzar un mayor grado de apertura y transparencia en las actuaciones realizadas por las universidades (UNESCO, 2009). En definitiva, las universidades deben ser socialmente responsables porque prestan un servicio público, como es la educación, y gestionan fondos públicos.

Proposición teórica $n^{\circ}$ 5: la comunicación y la colaboración con los grupos de interés es constante en la Universidad de Santiago de Compostela, en la Universidad Internacional de Andalucía y en la Universidad de Málaga, estableciendo dichas instituciones los mecanismos oportunos para garantizar dicha situación.

Los grupos de interés o stakeholders están presentes en la definición del concepto de Responsabilidad Social (Carroll, 1999; Schermerhorn, 2002). Por tanto, las universidades, al igual que otras organizaciones, deben considerar sus expectativas y necesidades, ya que la participación de los grupos de interés de la Universidad implica una concepción dialogada y plural de la Responsabilidad Social.

La Universidad de Santiago de Compostela, la Universidad Internacional de Andalucía y la Universidad de Málaga consideran, de manera significativa, a sus grupos de interés internos (personal docente e investigador, personal de administración y servicios, alumnos, etc.) y externos (proveedores, empresas, etc.), siguiendo las pautas establecidas, entre otros, por los Principios para la Educación Responsable en Gestión (ONU, 2007). En relación con los grupos de interés externos, destaca el diseño de estrategias de cooperación entre las universidades analizadas y las empresas, generando dichos acuerdos un mayor nivel de desarrollo de la sociedad.

Para finalizar, comentar que estas tres universidades están orientadas hacia la excelencia en todas sus actividades, tanto las clásicas docentes e investigadoras como las relativas a la satisfacción de sus grupos de interés, siendo ésta última la que las convierte en universidades excelentes (EFQM, 2010).

En este sentido, cabe destacar, que la Universidad de Málaga está comprometida con el logro de la excelencia, cuya consecución le exige garantizar la calidad en la totalidad de sus actividades. Al respeto, señalar lo siguiente: evaluación de la totalidad del Área de Servicios de la Universidad en el Modelo EFQM de Excelencia y obtención del Sello de Compromiso Europeo con la Excelencia 200+, otorgado por el Club Excelencia en Gestión. Vía Innovación y la Agencia Nacional de Evaluación de la Calidad y Acreditación (ANECA); el Secretariado de Deporte Universitario ha alcanzado el Sello de Excelencia Europea 300+, concedido a su sistema de gestión tras ser evaluado por las dos entidades referidas.

Por tanto, después del análisis realizado, se puede afirmar que las universidades objeto de estudio son gestionadas con criterios de Responsabilidad 
Social, porque identifican a sus grupos de interés y diseñan políticas basadas en la relación mantenida con los mismos (Vázquez, 2005). Además, la gestión de las relaciones que la Universidad de Santiago de Compostela, la Universidad Internacional de Andalucía y la Universidad de Málaga mantienen con sus grupos de interés las han adoptado de manera voluntaria, tal y como indican diversos autores especializados en la materia (Carroll y Shabana, 2010).

Proposición teórica $n^{\circ}$ 6: la existencia de una unidad organizativa encargada de la Responsabilidad Social en la Universidad de Santiago de Compostela, en la Universidad Internacional de Andalucía y en la Universidad de Málaga facilita el cumplimiento y la implantación de la misma en las citadas universidades. Dichas unidades utilizan diversos instrumentos, tales como, planes estratégicos, memorias de Responsabilidad Social o contratos programa para reforzar sus actuaciones en materia de Responsabilidad Social.

La información recabada, a través de los medios indicados con anterioridad, pone de manifiesto la existencia de un vicerrectorado especializado, órgano de gobierno unipersonal de máximo nivel, que centraliza las acciones sobre Responsabilidad Social en las instituciones de educación superior expuestas, lo que manifiesta una clara intención, por parte de las mismas, de garantizar la presencia de la Responsabilidad Social en las políticas que desarrollan. Esto coincide con lo reflejado en la literatura (Luzón et al., 2006), en la medida que la aplicación de criterios de Responsabilidad Social en la gestión universitaria supone desarrollar un comportamiento propio de las universidades socialmente responsables.

Para reforzar su compromiso con la Responsabilidad Social, la Universidad Santiago de Compostela, la Universidad Internacional de Andalucía y la Universidad de Málaga, emplean diversos instrumentos de gestión universitaria, tales como, planes estratégicos, contratos programa, cuadros de mando, códigos de conducta, entre otros. En consonancia con lo expuesto, cabe destacar, que las Memorias de Responsabilidad Social son una herramienta fundamental dentro de los procesos de información, constituyendo además, un instrumento clave para conseguir una mejor comunicación con los diferentes grupos de interés (Del Pozo et al, 2008).

Finalmente, indicar que el creciente número de memorias publicadas por diversas organizaciones, entre las que se encuentran un número significativo de universidades, pone de manifiesto la importancia, que, en la actualidad, presentan las mismas (Albareda y Balaguer, 2008).

Proposición teórica $n^{\circ}$ 7: la Universidad de Santiago de Compostela, la Universidad Internacional de Andalucía y la Universidad de Málaga, a través de sus respectivos compromisos con la Responsabilidad Social, han obtenido mejores resultados en materia social, medioambiental y económica. Además, la relación con sus grupos de interés es más fluida y existe mayor colaboración con los mismos.

La Universidad de Santiago de Compostela, la Universidad Internacional de Andalucía y la Universidad de Málaga conocen los impactos que generan, al desarrollar sus actividades, en el entorno. El compromiso asumido por estas institucio- 
nes con la eliminación o reducción de dichos impactos, o lo que es lo mismo, la participación en actividades relativas a la Responsabilidad Social genera, según McWilliams et al. (2006), un efecto positivo en sus grupos de interés. Por tanto, las actuaciones desarrolladas, en los tres ámbitos (social, medio ambiental y económico) que definen a la Responsabilidad Social, han dado lugar a la creación de efectos positivos en sus grupos de interés, lo que, según Marín y Rubio (2008), constituye la base del éxito de la Responsabilidad Social.

En concreto, las universidades estudiadas, representativas del Sistema Universitario Español, se pueden catalogar como socialmente responsables. Sin embargo, la ausencia de estándares de gestión universitaria en materia de Responsabilidad Social, ampliamente consolidados, origina que cada institución la incorpore en sus estrategias de manera diferente. Asimismo, se aprecia como la consideración de la dimensión social, que constituye un eje estratégico en la gestión de las unidades de análisis, ocasiona mejoras en el conjunto de las universidades, contribuyendo, de manera destacada, a mejorar las relaciones con sus grupos de interés y el entorno en el que desarrollan sus actividades.

En cualquier caso, la gestión de la Responsabilidad Social que llevan a cabo las instituciones analizadas constituye una referencia relevante para el resto de universidades y centros de enseñanza superior de España, ya que pone de manifiesto la capacidad que tiene la Universidad Española para influir, de manera destacada, en los acontecimientos sociales.

\section{Conclusiones}

La Responsabilidad Social ha adquirido, en los últimos años, una relevancia clave en la gestión de las organizaciones. Hasta ahora, la literatura, en la mayoría de los casos, se ha ocupado de estudiar dicha materia en el ámbito empresarial. Sin embargo, el enfoque que adoptado en el presente trabajo de investigación centró la atención en otro tipo de organización, la Universidad.

Para ello, se escogieron, como unidades de análisis, tres instituciones pertenecientes al Sistema Universitario Español: la Universidad de Santiago de Compostela, la Universidad Internacional de Andalucía y la Universidad de Málaga. La aplicación de técnicas de investigación cualitativas se han considerado oportunas porque, en términos generales, aportan resultados satisfactorios cuando se estudian fenómenos complejos.

Este trabajo ha llevado a la obtención de conclusiones generales que permitirán avanzar en la investigación sobre la Responsabilidad Social Universitaria. Entre ellas, destaca que las instituciones estudiadas han incorporado a sus sistemas de gestión, en consonancia con el auge de la dimensión social de la educación superior, los principios que definen a la Responsabilidad Social, lo que ha supuesto mejoras significativas en las propias instituciones y en las relaciones con sus grupos de interés. Asimismo, las universidades, al igual que otras organizaciones, generan, al realizar sus actividades, impactos en su entorno, siendo un objetivo prioritario para las mismas eliminar o reducir aquellos que sean negativos. 
En definitiva, el trabajo presentado pone de manifiesto que las universidades analizadas asumen un compromiso, serio y constante, con la sociedad, contribuyendo así a la mejora y al progreso de la comunidad en la que realizan sus actividades. Estas organizaciones, por consiguiente, son agentes fundamentales para avanzar en la recuperación económica y reforzar la cohesión social. La consideración de la Responsabilidad Social en la gestión universitaria, por parte de las universidades analizadas, refleja el compromiso de estas instituciones con la mejora del entorno, el desarrollo sostenible y la excelencia en el desempeño de sus actividades.

Además, la creación, por parte de las instituciones referidas, de unidades organizativas específicas encargadas de la Responsabilidad Social y el desarrollo de diferentes instrumentos de gestión universitaria son hechos significativos que muestran el interés de las mismas por transmitir a sus grupos de interés los resultados que obtienen al realizar todas sus actividades.

Aunque la incorporación de la Responsabilidad Social difiere en algunos aspectos, como el relativo a las certificaciones medioambientales o los mecanismos de comunicación con sus grupos de interés, las tres universidades consideran los aspectos económicos, sociales y medioambientales, en un contexto de voluntariedad, en su gestión, siendo, por tanto, universidades socialmente responsables. Así, se constata que la Responsabilidad Social responde a la relación existente entre las actividades que desempeñan las universidades y lo que el entorno espera de ellas, adquiriendo un carácter estratégico la función de servicio a la sociedad.
Finalmente, no se pueden obviar las limitaciones que presenta la investigación realizada. Aunque este trabajo demuestra que la metodología del estudio de casos puede resultar adecuada para el análisis exploratorio de nuevos modelos de gestión en las instituciones de educación superior, presenta una limitación propia de las investigaciones cualitativas, que es no poder generalizar los resultados en términos estadísticos. Además, otra limitación es que las universidades objeto de estudio han sido seleccionadas entre un gran número de ellas. Ello debe evitarse en investigaciones posteriores, siempre que a partir de las conclusiones obtenidas en el presente trabajo se formulen hipótesis concretas a contrastar en una muestra amplia de universidades.

\section{Referencias Bibliográficas}

Albareda Vivó, Laura y Balaguer Franch, María Rosario (2008). La responsabilidad social de la empresa y los resultados financieros. Revista de Contabilidad y Dirección, No. 7, pp. 11-26.

Aldeanueva Fernández, Ignacio (2012). Aplicación de la metodología del análisis de casos al estudio de la Responsabilidad Social Universitaria. Documento presentado en el IX Foro Internacional sobre la Evaluación de la Calidad de la Investigación y de la Educación Superior. Santiago de Compostela.

Aras, Guler, Aybars, Asli y Kutlu, Ozlem (2010). Managing corporate performance. Investigating the relationship between corporate social responsibility and financial performance in emerging markets. International Journal of Productivity and Performance Management, Vol. 59, No. 3, pp. 229-254. 
Bañón, Alexis, Guillén, Manuel y Ramos, Natalia (2011). La Empresa Ética y Responsable. Universia Business Review, No. 30, pp. 32-43.

Benneworth, Paul y Jongbloed, Ben W. (2010). Who matters to universities? A stakeholder perspective on humanities, arts and social sciences valorization. Higher Education, Vol. 59, No. 5, pp. 567-588.

Brito, Morelba (2007). Gobernanza empresarial: ética, responsabilidad social y rentabilidad en la "Era pos-Enron". Revista Venezolana de Gerencia, Vol. 12, No. 38, pp. 183-206.

Carroll, Archie B. (1999). Corporate social responsibility: evolution of a definitional construct. Business \& Society, Vol. 38, No. 3, pp. 268-295.

Carroll, Archie B. y Shabana, Kareem M. (2010). The business case for corporate social responsibility: a review of concepts, research and practice. International Journal of Management Reviews, Vol. 12, No.1, pp. 85-105.

De la Red Vega, Natividad (2009). Necesidades emergentes y responsabilidad social universitaria. Alternativas. Cuadernos de Trabajo Social, No. 16, pp. 65-76.

Del Pozo, Raúl, Cuesta, Cristina y Roca, Francisco. (2008). La verificación de la memoria como elemento de gestión de la responsabilidad corporativa de la empresa. Revista de Contabilidad y Dirección, No. 7, pp. 219- 236.

DHO (2007). Project AISHE. Stichting Duurzaam Hoger Onderwijs. Ámsterdam.

Días, Marco Antonio (2008). La universidad en el siglo XXI: del conflicto al diálogo de civilizaciones. Educación Superior y Sociedad, No. 2, pp. 91-138.

EFQM (2010). Modelo EFQM de Excelencia 2010. Fundación Europea para la Gestión de la Calidad. Bruselas.
EUA (2005). Declaración de Glasgow. Universidades fuertes para una Europa fuerte. Asociación de Universidades Europeas. Bruselas.

EUA (2007). Declaración de Lisboa. Las universidades de Europa más allá de 2010: diversidad con un propósito común. Asociación de Universidades Europeas. Bruselas.

Freeman, Robert Edward (1984). Strategic Management: A stakeholder approach. Boston, Pitman.

Husted, Bryan W. (2000). A contingency theory of corporate social performance. Business \& Society, Vol. 39, No.1, pp. 24-48.

Jiménez de la Jara, Mónica (2008). ¿Cómo medir la percepción de la responsabilidad social en los diversos estamentos de la universidad? Una experiencia concreta. Educación Superior y Sociedad, No. 2, pp. 139-162.

Jin, K. Gregory y Drozdenko, Ronald G. (2010). Relationships among perceived organizational core values, corporate social responsibility, ethics and organizational performance outcomes: an empirical study of information technology professionals. Journal of Business Ethics, Vol. 92, No. 3, pp. 341-359.

Luzón Trujillo, Antonio, Pereyra, Miguel A. y Sevilla Merino, Diego (2006). Las universidades españolas y el proceso de construcción del Espacio Europeo de Educación Superior. Limitaciones y perspectivas de cambio. Revista Española de Educación Comparada, No. 12, pp. 113-143.

Maon, François, Lindgreen, Adam y Swaen, Valérie (2010). Organizational stages and cultural phases: a critical review and consolidative model of corporate social responsibility development. International Journal of Management Reviews, Vol. 12 , No. 1, pp. 20-38. 
Marín Rives, Longinos y Rubio Bañón, Alicia (2008). ¿Moda o factor competitivo? Un estudio empírico de responsabilidad social corporativa en pyme. Información Comercial Española, Revista de Economía, No. 842, pp. 177-193.

Martínez de Carrasquero, Cynthia, Rojas de Mavárez, Ligibther, Guillén Romero, Jaqueline y Antúnez, Nereida (2012). Responsabilidad social universitaria, transferencia y mercadeo tecnológico: vinculación con el entorno social. Revista Venezolana de Gerencia, Vol. 17, No. 59, pp. 512-528.

Matten, Dirk y Moon, Jeremy (2008). Implicit and explicit CSR: a conceptual framework for a comparative understanding of corporate social responsibility. Academy of Management Review, Vol. 33, No. 2, pp. 404-424.

McWilliams, Abagail; Siegel, Donald S. y Wright, Patrick M. (2006). Corporate social responsibility: strategic implications. Journal of Management Studies, Vol. 43, No. 1, pp. 1-18.

Ministerio de Ciencia e Innovación (2009). Estrategia Universidad 2015. Universidades para el progreso, el bienestar y la competitividad. Madrid.

NJHEPS (2004). High Performance Campus. Design Handbook. Volume 1: Overview and Rationale. New Jersey Higher Education Partnership for Sustainability. Nueva Jersey.

Noland, James y Phillips, Robert (2010). Stakeholder engagement, discourse ethics and strategic management. International Journal of Management Reviews, Vol. 12, No. 1, pp. 39-49.

ONU (2007). Principios para la Educación Responsable en Gestión (PRME). Organización de las Naciones Unidas. Nueva York.

Porter, Michael E. y Kramer, Mark R. (2006). Strategy \& Society. The link between competitive advantage and corporate social responsibility. Harvard Business Review, Vol. 84, No. 2, pp. 7892.

Schermerhorn, John R. (2002). Management (séptima edición). Nueva York, Wiley.

Schlusberg, Malcolm D. (1969). Corporate legitimacy and social responsibility: the role of law. California Management Review, Vol. 12, No. 1, pp. 65-76.

Schwartz, Birgitta y Tilling, Karina (2009). ISO-lating corporate social responsibility in the organizational context: a dissenting interpretation of ISO 26000. Corporate Social Responsibility and Environmental Management, Vol. 16, No. 5, pp. 289-299.

Siracusa, Giuseppe, Ardiles, Arlene y Pulido, Sirse (2012). Gerencia universitaria postmoderna en el paradigma de la responsabilidad social. Revista Venezolana de Gerencia, Vol. 17, No. 58, pp. 307-320.

UNESCO (2007). Los dos primeros años del Decenio de las Naciones Unidas de la Educación para el Desarrollo Sostenible (2005-2014). Organización de las Naciones Unidas para la Educación, la Ciencia y la Cultura. París.

UNESCO (2009). Conferencia Mundial sobre la Educación Superior: la nueva dinámica de la educación superior y la investigación para el cambio social y el desarrollo. Organización de las Naciones Unidas para la Educación, la Ciencia y la Cultura. París.

Urdaneta, Mary Josefina (2008). La responsabilidad social en la industria farmacéutica del estado Zulia. Revista Venezolana de Gerencia, Vol. 13, No. 41, pp. 51-74.

Vallaeys, François (2008). Responsabilidad social universitaria: una nueva filosofía de gestión ética e inteligente para las universidades. Educación Superior y Sociedad, No. 2, pp. 191-220. 
Responsabilidad social universitaria en España: un estudio de casos

Aldeanueva F., Ignacio y Jiménez Q., José Antonio

Vázquez Oteo, Orencio (2005). La Responsabilidad social corporativa: el papel de la sociedad civil. Ciriec-España, Revista de Economía Pública, Social y Cooperativa, No. 53, pp. 111-124.
Yin, Robert K. (1989). Case Study Research: design and methods. Applied Social Research Methods Series. Newbury Park (California), Sage Publications. 